\title{
Aplicaciones móviles para el cuidado respiratorio del neonato, revisión narrativa
}

\section{Mobile applications for newborn respiratory care, narrative review}

\author{
Janeth Carrillo-Franco iD', Angie Paola Avella-Charrasquiel (iD², Helver Fabián López-Vargas (iD)
}

1. Fundación Universitaria del Área Andina. Bogotá, Colombia. Correo: jcarrillo27@areandina.edu.co - https://orcid.org/0000-0002-9957-6995.

2. Fundación Universitaria del Área Andina. Bogotá, Colombia. Correo: aavella3@estudiantes.areandina.edu.co - https://orcid.org/0000-0003-07906190.

3. Fundación Universitaria del Área Andina. Bogotá, Colombia. Correo: hlopez25@estudiantes.areandina.edu.co - https://orcid.org/0000-0002-0778873x.

Tipología: Artículo de revisión

Para citar este artículo: Carrillo-Franco J, Avella-Charrasquiel A, López-Vargas, F. Aplicaciones móviles para el cuidado respiratorio del neonato, revisión narrativa. Duazary. 2021 abril; 18(2): 188-198. Doi: https://doi.org/10.21676/2389783X.4076

\section{Palabras clave: neonato; cuidado del niño; salud respiratoria; telemedicina; cuidadores.}

\section{RESUMEN}

El cuidado respiratorio del recién nacido es una de las actividades más importantes para su adaptación, ya que el bebé debe ajustar su respiración al pasar del medio líquido al ambiente. Así, su esfuerzo respiratorio será decisivo para garantizar el paso funcional de la vida intrauterina a la extrauterina. En el periodo neonatal, es importante que los padres puedan detectar signos de alarma respiratorio, y las tecnologías en comunicación son una de las herramientas para acercar la información en tiempo real a los cuidadores. Este artículo describe una revisión narrativa de literatura para identificar aplicaciones móviles sobre cuidado respiratorio del neonato, en la cual se utilizaron términos del MeSH: "infant newborn", "child care", "respiratory therapy", "telemedicine", "eHealth", "caregivers" y sus correspondientes del DeCS (español y portugués). Se estableció un protocolo de búsqueda en bases de datos suscritas y libres, que incluyó filtros de completitud y calidad de los artículos. Se encontraron 488 artículos, a los cuales se aplicaron criterios de inclusión y calidad. De este modo quedaron 19 artículos completos para lectura y análisis. A pesar del diseño creciente de aplicaciones en salud, en el área del cuidado respiratorio del neonato no se encontraron aplicaciones diseñadas para tal fin o que fueran específicas para cuidadores.

\section{ABSTRACT}

Keywords: infant newborn; childcare; respiratory therapy; telemedicine; caregivers.
Respiratory care of the newborn is one of the most important activities for his adaptation, since the baby must adjust his breathing when moving from the liquid medium to the environment, where his respiratory effort will be decisive in guaranteeing the functional passage from intrauterine life to extrauterine. In the neonatal period, detection of respiratory warning signs by parents is important, and communication technologies are one of the tools to bring information in real time to caregivers. This article describes a narrative literature review to identify mobile applications on newborn respiratory care, in which MeSH terms were used: infant newborn, childcare, respiratory therapy, telemedicine, eHealth, caregivers and their corresponding DeCS (Spanish and Portuguese). A search protocol in subscribed and free databases was established, that included filters of completeness and quality of the articles. 488 articles were found, to which inclusion and quality criteria were applied, leaving 19 complete articles for reading and analysis. Despite the increasing design of health applications, in the area of neonatal respiratory care, no applications were found that were designed for this purpose and that were specific for caregivers. 


\section{INTRODUCCIÓN}

El cuidado respiratorio del neonato es una de las actividades más importantes para su adaptación. Al nacer, el bebé debe adaptar su respiración al pasar del medio líquido en el que se ha desarrollado al ambiente, donde su esfuerzo respiratorio será decisivo para garantizar el paso funcional de la vida intrauterina a la extrauterina ${ }^{1}$.

Tanto los recién nacidos a término (después de cumplir 37 semanas de gestación) como los pretérminos pueden presentar diversas afectaciones respiratorias de gravedad diversa. En los segundos, tales afectaciones pueden ser derivadas principalmente de su prematurez pulmonar ${ }^{2}$. Para ambos grupos, los problemas respiratorios constituyen una importante causa de mortalidad y morbilidad en el neonato.

El síndrome de dificultad respiratoria del recién nacido (SDR) se relaciona de manera inversa con la edad gestacional y con el peso al nacer ${ }^{3}$. Esto es, su presentación en el neonato a término es probable pero no siempre ocurre, y los problemas respiratorios de este grupo pueden estar asociados a la dificultad de reabsorción del líquido por el pulmón, la asfixia perinatal, las infecciones respiratorias (neumonía neonatal) o trastornos de la circulación pulmonar (hipertensión pulmonar) ${ }^{4}$. Sin embargo, la mayoría de neonatos a término no experimentan dificultades respiratorias, y uno de los objetivos de los servicios de salud es minimizar las actividades intervencionistas sobre el recién nacido sano y disminuir su estancia hospitalaria para que pueda darse de alta con sus padres/cuidadores pocas horas después del parto ${ }^{5}$.

Si bien existen guías basadas en la evidencia con recomendaciones para padres de recién nacidos sanos sobre el manejo de los principales problemas que pueden presentarse en el neonato sin factores de riesgo ${ }^{6}$, ciertos aspectos del cuidado respiratorio y de signos de alarma específicos requieren información permanente como actividades de educación y promoción de la salud ${ }^{7}$.
Algunas características de los cuidadores se han asociado con dificultad en el reconocimiento temprano de signos de alarma respiratoria en el neonato. Domínguez-Anaya et $a l^{8}$ señalan que determinantes personales como edad (adolescentes) y nivel educativo bajo impactan de forma negativa en los conocimientos de las madres sobre los signos de alarma de infección respiratoria aguda en niños. Otros estudios han descrito que la probabilidad de solicitar atención médica para problemas respiratorios es menor en familias que viven en condiciones de pobreza, de madres muy jóvenes o residentes a mayor distancia de los servicios clínicos ${ }^{9}$. También se ha propuesto que el conocimiento del cuidador podría ser un factor pronóstico de la infección respiratoria en el niño ${ }^{10}$.

Para acercar la información en tiempo real a los padres y cuidadores, las tecnologías en comunicación son un importante aliado de la salud, y su uso presenta una tendencia en aumento en el mundo. Se espera que estas herramientas sirvan para la actividad asistencial de los profesionales de salud y para el usuario como medio de empoderamiento a través de la información. Así, Abaza y Marscholleck ${ }^{11}$, en su revisión sistemática de literatura, reportan 842 artículos analizados sobre aplicaciones móviles y su uso en salud ${ }^{12}$, la mayoría de las cuales se dirigen al control de los pacientes crónicos, a la cesación del tabaquismo y al aumento de la actividad física. En el área de salud materna e infantil, una revisión sistemática evaluó 76 aplicaciones comerciales para las gestantes, y encontró que aquellas con presentaciones atractivas y fáciles de leer eran las más usadas.

El objetivo de este artículo es presentar una revisión narrativa de literatura sobre aplicaciones móviles a partir de la pregunta de investigación ¿Qué aplicaciones para dispositivos móviles reporta la literatura, diseñadas para que el cuidador reconozca los signos de alarma respiratorios en neonatos y cuente con información sobre cuidado y promoción de su salud respiratoria?

\section{MATERIALES Y MÉTODOS}


Esta revisión narrativa hizo parte del proyecto "Tecnología y cuidado prenatal comunitario en áreas vulnerables de Girardot", como contribución en la identificación de aplicaciones móviles diseñadas para el cuidado respiratorio del recién nacido.

\section{Protocolo de búsqueda}

Búsqueda de documentos: se realizó una revisión general de la literatura según descriptores seleccionados en bases de datos de acceso libre (Scielo, Redalyc, PubMed, BMC) y suscritas (Science Direct, ProQuest, Gale), identificando artículos que cumplieran criterios de inclusión. A estos se realizó lectura de resúmenes.

Descriptores seleccionados: se seleccionaron los siguientes términos del MeSH: "infant newborn", "child care", "respiratory therapy", "telemedicine", "eHealth", "caregivers" y sus correspondientes del DeCS en español: "recién nacido", "cuidado del niño", "salud respiratoria", "mSalud", "telemedicina", y en portugués: "cuidadores", "recem nascido", "cuidado da criança", "terapia respiratória", "telemedicina", "cuidadores".

\section{Criterios de inclusión}

- Ventana temporal del 2014 al 2019.

- Artículos en inglés, español y portugués.

- Artículos sobre aplicaciones móviles usadas para el cuidado respiratorio del neonato.

- Artículos referentes a estudios experimentales y observacionales y revisiones sistemáticas de literatura.

\section{Selección de documentos}

Se tomaron aquellos artículos de interés que cumplieran con los criterios arriba mencionados y se registraron en la matriz diseñada para tal fin, que incluía un primer filtro de bases y un segundo de calidad y completitud. Este último filtro incluyó las preguntas “¿Qué objetivo tuvo el estudio?”, “¿Cuál fue la metodología utilizada?”, “¿Qué resultados reportan?" y "¿Qué desafíos identificaron?". Los artículos que cumplían el segundo filtro se descargaron para lectura completa y análisis en las categorías descritas.

\section{RESULTADOS}

La búsqueda en las siete bases de datos seleccionadas arrojó un total de 488 artículos iniciales y 104 de otras fuentes, de los cuales se eliminaron duplicados. De este modo se obtuvo un total de 68 artículos del primer filtro. Luego de leer los resúmenes y al aplicar los criterios de selección, se eligieron 19, los cuales se leyeron en texto completo (figura 1). Cuatro de estos artículos se excluyeron por incompletos, ya que no evaluaban directamente la aplicación ni sus usos.

La Tabla 1 describe las principales características de los 19 artículos incluidos, para los cuales se analizó población a la que se dirige, objetivos del estudio y resultados más relevantes desde el punto de vista de la revisión sobre cuidado respiratorio. 


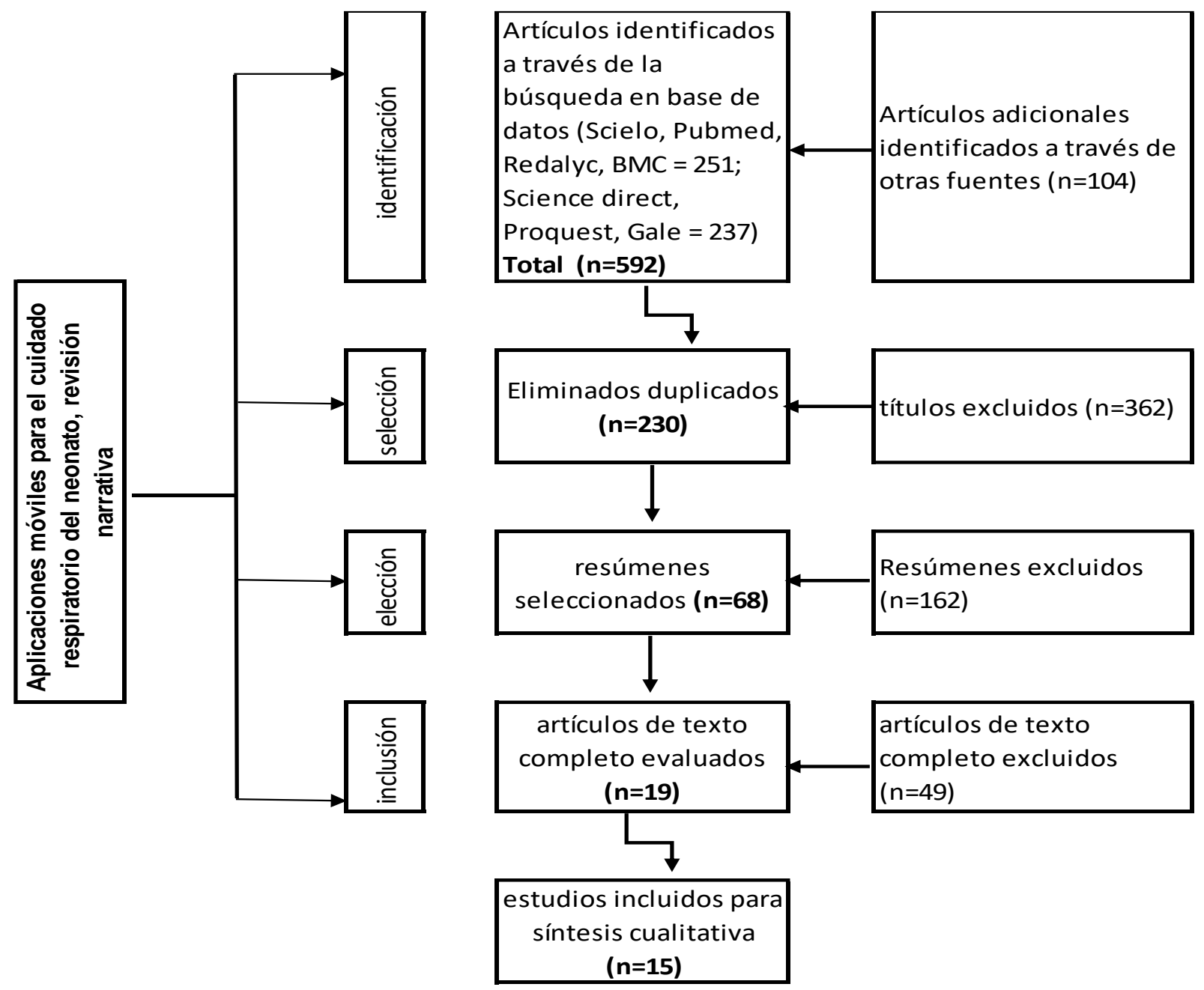

Figura 1. Diagrama de flujo de búsqueda y selección de artículos. 
Tabla 1. Características de artículos incluidos.

\begin{tabular}{|c|c|c|c|c|}
\hline Categoría & Referencia & $\begin{array}{l}\text { Población objeto } \\
\text { de la aplicación }\end{array}$ & Tipo u objetivo del estudio & Resultados/desafíos \\
\hline $\begin{array}{l}\text { Atención } \\
\text { prenatal }\end{array}$ & $\begin{array}{l}\text { Van den } \\
\text { Heuvel et } \\
a l^{13}\end{array}$ & $\begin{array}{l}\text { Aplicaciones para } \\
\text { mujeres en } \\
\text { atención prenatal }\end{array}$ & $\begin{array}{l}\text { Revisión de literatura sobre uso de } \\
\text { mSalud en el embarazo. }\end{array}$ & $\begin{array}{l}\text { Las intervenciones de mSalud tienen un campo de } \\
\text { aplicación multinivel centrado en la atención } \\
\text { perinatal. }\end{array}$ \\
\hline $\begin{array}{l}\text { Atención } \\
\text { prenatal }\end{array}$ & Halili et $a l^{14}$ & Mujeres gestantes & $\begin{array}{l}\text { Evaluación de la receptividad y } \\
\text { funcionalidad de la aplicación } \\
\text { SmartMoms Canada mHealth. }\end{array}$ & $\begin{array}{l}\text { Las mujeres valoraron positivamente el potencial de } \\
\text { la aplicación y ofrecieron comentarios para mejorar } \\
\text { la próxima versión. }\end{array}$ \\
\hline $\begin{array}{l}\text { Atención } \\
\text { prenatal, } \\
\text { posparto y } \\
\text { del menor de } \\
\text { un año }\end{array}$ & $\begin{array}{l}\text { Chowdhury } \\
\text { et } a l^{15}\end{array}$ & $\begin{array}{l}\text { Mujeres } \\
\text { embarazadas y } \\
\text { nuevas madres }\end{array}$ & $\begin{array}{l}\text { Estudio observacional para evaluar el } \\
\text { efecto del uso de Aponjon (servicio } \\
\text { mHealth basado en teléfonos móviles) } \\
\text { de atención de la salud materna y } \\
\text { neonatal. }\end{array}$ & $\begin{array}{l}\text { El uso de la aplicación por lo menos durante seis } \\
\text { meses fue asociado con aumento del conocimiento } \\
\text { sobre las prácticas de cuidado. }\end{array}$ \\
\hline $\begin{array}{l}\text { Atención a } \\
\text { mujeres } \\
\text { posparto }\end{array}$ & $\begin{array}{l}\text { Mbuthia et } \\
a^{16}\end{array}$ & Mujeres posparto & $\begin{array}{l}\text { Revisión sistemática de literatura de } \\
\text { artículos sobre la comunicación de } \\
\text { mHealth para fortalecer la atención } \\
\text { posnatal en las zonas rurales. }\end{array}$ & $\begin{array}{l}\text { La revisión muestra que la mensajería unidireccional } \\
\text { del teléfono móvil es el tipo común de comunicación } \\
\text { mHealth utilizada para fortalecer la atención } \\
\text { posnatal en las zonas rurales. }\end{array}$ \\
\hline $\begin{array}{l}\text { Atención de } \\
\text { mujeres y } \\
\text { recién nacido }\end{array}$ & $\begin{array}{l}\text { Prinja et } \\
a f^{17}\end{array}$ & $\begin{array}{l}\text { Voluntarios de } \\
\text { salud comunitaria } \\
\text { para la atención de } \\
\text { mujeres gestantes y } \\
\text { recién nacidos }\end{array}$ & $\begin{array}{l}\text { Evaluar el impacto de una aplicación } \\
\text { de salud móvil en el proyecto } \\
\text { "Reducción de las muertes maternas y } \\
\text { del recién nacido (ReMiND) en India". }\end{array}$ & $\begin{array}{l}\text { Tres de los ocho servicios registraron una mejora } \\
\text { significativa como resultado de la intervención de } \\
\text { salud móvil (suplementación de ácido fólico, } \\
\text { autorreporte de complicaciones y dudas posparto). }\end{array}$ \\
\hline $\begin{array}{l}\text { Atención } \\
\text { prenatal e } \\
\text { infantil }\end{array}$ & $\begin{array}{l}\text { Willcox et } \\
a 1^{18}\end{array}$ & $\begin{array}{l}\text { Trabajadores de la } \\
\text { salud }\end{array}$ & $\begin{array}{l}\text { Pronosticar la rentabilidad incremental } \\
\text { de la iniciativa Tecnología móvil para la } \\
\text { Salud Comunitaria (MOTECH) a escala } \\
\text { en } 170 \text { distritos de Ghana. }\end{array}$ & $\begin{array}{l}\text { La adopción de MOTECH para mejorar la prestación } \\
\text { y la captación de servicios de salud materna e } \\
\text { infantil representa una buena relación calidad- } \\
\text { precio. }\end{array}$ \\
\hline $\begin{array}{l}\text { Atención en } \\
\text { salud } \\
\text { materna, } \\
\text { neonatal e } \\
\text { infantil }\end{array}$ & Lee et $a l^{19}$ & $\begin{array}{l}\text { Aplicaciones } \\
\text { diseñadas para } \\
\text { intervenciones en } \\
\text { salud materna, } \\
\text { neonatal e infantil }\end{array}$ & $\begin{array}{l}\text { Revisión de literatura para evaluar la } \\
\text { efectividad de las intervenciones de } \\
\text { mHealth para la salud materna, } \\
\text { neonatal e infantil en países de bajos y } \\
\text { medianos ingresos. }\end{array}$ & $\begin{array}{l}\text { La mayoría de los estudios de } m \text { Health son de baja } \\
\text { calidad metodológica, y pocos evalúan los impactos } \\
\text { en los resultados de los pacientes. }\end{array}$ \\
\hline $\begin{array}{l}\text { Atención } \\
\text { materna e } \\
\text { infantil }\end{array}$ & $\begin{array}{l}\text { Martínez- } \\
\text { Fernández } \\
\text { et } a l^{20}\end{array}$ & $\begin{array}{l}\text { Facilitadores } \\
\text { comunitarios de } \\
\text { salud }\end{array}$ & $\begin{array}{l}\text { Describir los resultados del uso de la } \\
\text { estrategia mHealth, basada en la } \\
\text { provisión de un teléfono celular a los } \\
\text { facilitadores de la comunidad, en } \\
\text { Guatemala. }\end{array}$ & $\begin{array}{l}\text { El proyecto ha demostrado una disminución } \\
\text { estadísticamente significativa en la mortalidad } \\
\text { materna }(p<0,05) \text { y en la mortalidad infantil } \\
(p=0,054) .\end{array}$ \\
\hline $\begin{array}{l}\text { Atención } \\
\text { prenatal y del } \\
\text { recién nacido }\end{array}$ & $\begin{array}{l}\text { Bakibinga } \\
\text { et } a p^{21}\end{array}$ & $\begin{array}{l}\text { Voluntarios } \\
\text { comunitarios de } \\
\text { salud }\end{array}$ & $\begin{array}{l}\text { Evaluar el valor agregado del uso de } \\
\text { teléfonos inteligentes para identificar y } \\
\text { rastrear a madres y niños por } \\
\text { voluntarios de salud comunitaria en } \\
\text { Nairobi, Kenia. }\end{array}$ & $\begin{array}{l}\text { Se diseñó una aplicación mHealth que utiliza } \\
\text { soluciones dinámicas de teléfono móvil y portal web } \\
\text { para permitir que los voluntarios comunitarios } \\
\text { tomen decisiones oportunas de atención en salud. }\end{array}$ \\
\hline $\begin{array}{l}\text { Atención en } \\
\text { salud }\end{array}$ & $\begin{array}{l}\text { Vialart } \\
\text { Vidal et a }{ }^{22}\end{array}$ & $\begin{array}{l}\text { Aplicaciones usadas } \\
\text { en salud }\end{array}$ & $\begin{array}{l}\text { Estudio descriptivo sobre los logros y } \\
\text { los desafíos de la aplicación de la } \\
\text { eSalud en el contexto cubano. }\end{array}$ & $\begin{array}{l}\text { Los proyectos de la eSalud cubana son modelos } \\
\text { avanzados de solidaridad, en los que se comparten } \\
\text { información y conocimiento. }\end{array}$ \\
\hline $\begin{array}{l}\text { Atención } \\
\text { neonatal }\end{array}$ & $\begin{array}{l}\text { Matin et } \\
a^{23}\end{array}$ & $\begin{array}{l}\text { Madres y mujeres } \\
\text { posparto }\end{array}$ & $\begin{array}{l}\text { Desarrollo de un dispositivo de } \\
\text { evaluación de la salud neonatal } \\
\text { (aplicación para teléfonos inteligentes } \\
\text { y un sensor portátil) para reconocer } \\
\text { signos de enfermedad neonatal. }\end{array}$ & $\begin{array}{l}\text { El } 64 \% \text { de las madres que recibieron una alerta del } \\
\text { sistema lo utilizaron de inmediato ante signos de } \\
\text { peligro. }\end{array}$ \\
\hline $\begin{array}{l}\text { Atención de } \\
\text { recién } \\
\text { nacidos } \\
\text { prematuros }\end{array}$ & $\begin{array}{l}\text { Sgandurra } \\
\text { et }\left.a\right|^{24}\end{array}$ & $\begin{array}{l}\text { Cuidadores con } \\
\text { recién nacidos } \\
\text { prematuros }\end{array}$ & $\begin{array}{l}\text { Evaluar, a través de un estudio de } \\
\text { ensayo clínico aleatorizado (ECA), los } \\
\text { efectos de la intervención de CareToy } \\
\text { en el desarrollo motor y visual } \\
\text { temprano en recién nacidos } \\
\text { prematuros. }\end{array}$ & $\begin{array}{l}\text { El sistema CareToy puede proporcionar una } \\
\text { intervención temprana (IE) efectiva en el hogar. }\end{array}$ \\
\hline $\begin{array}{l}\text { Atención de } \\
\text { recién } \\
\text { nacidos } \\
\text { prematuros o } \\
\text { enfermos } \\
\end{array}$ & $\begin{array}{l}\text { Schaeffer et } \\
a^{25}\end{array}$ & $\begin{array}{l}\text { Trabajadores de } \\
\text { salud comunitarios } \\
\text { en atención de } \\
\text { recién nacidos }\end{array}$ & $\begin{array}{l}\text { Evaluar el potencial de la tecnología } \\
\text { móvil de salud para mejorar el } \\
\text { diagnóstico y el manejo de casos de } \\
\text { enfermedades prevalentes del recién } \\
\text { nacidos. }\end{array}$ & $\begin{array}{l}\text { Los trabajadores comunitarios de salud con poca o } \\
\text { ninguna experiencia en el uso de tabletas móviles } \\
\text { pueden usar y operar una aplicación móvil para } \\
\text { evaluaciones de recién nacidos. }\end{array}$ \\
\hline $\begin{array}{l}\text { Atención del } \\
\text { recién nacido } \\
\text { sano }\end{array}$ & $\begin{array}{l}\text { Cervantes } \\
\text { Guijarro et } \\
a^{26}\end{array}$ & $\begin{array}{l}\text { Cuidadores de } \\
\text { recién nacidos } \\
\text { sanos }\end{array}$ & $\begin{array}{l}\text { Estudio piloto de intervención para } \\
\text { seguimiento, a través de web, redes } \\
\text { sociales y telemedicina, de las } \\
\text { revisiones del niño sano de } 1-2,2-4 \text { y } \\
\text { 4-6 meses. }\end{array}$ & $\begin{array}{l}\text { La telemedicina es eficaz en control, seguimiento y } \\
\text { prevención de problemas habituales del lactante } \\
\text { sano. }\end{array}$ \\
\hline
\end{tabular}




\section{DISCUSIÓN}

Las aplicaciones diseñadas para dispositivos móviles (apps) hacen parte de las tecnologías de la información y las comunicaciones (TIC) y le brindan al usuario información en tiempo real. En los últimos años, la producción de aplicaciones móviles para el acceso a contenido ha aumentado de manera logarítmica en su distribución y consumo, en ámbitos diversos como la educación, el entretenimiento, el turismo, la salud, la práctica de actividad física, entre otros ${ }^{28-31}$. Concretamente en el campo de la salud, su diseño y su uso muestran una tendencia en crecimiento ${ }^{32}$. De este modo se ha acogido el término "mSalud" (eHealth en inglés) para designar el uso de TIC en salud ${ }^{33}$.

Según Kao y Liebovitz ${ }^{34}$, en un estudio que tuvo como objetivo realizar un inventario de apps de salud, en 2017 existían más de 165.000 aplicaciones móviles de salud de acceso público, la gran mayoría diseñadas para pacientes, diferenciadas por los autores en dos grandes categorías: una relacionada con el control de bienestar y de enfermedades, y la segunda tiene que ver con autodiagnóstico, recordatorio de medicamentos y acceso a proveedores de salud.

En esta amplia oferta se encuentran aplicaciones relacionadas con el control específico de enfermedades. Por ejemplo, en una revisión sistemática sobre apps para enfermedad de Parkinson se encontraron 125 aplicaciones relacionadas, 56 con potencial utilidad, 69 con un diseño específico para la enfermedad, 23 de información, 29 de valoración, 13 de tratamiento y 4 de valoración y tratamiento ${ }^{35}$. Sin embargo, existe también preocupación creciente por la brecha digital entre los ciclos vitales y los grupos económicamente vulnerables, ya que personas mayores o personas sin recursos tienen dificultad para acceder a móviles inteligentes $\mathrm{y} / \mathrm{o}$ al uso de $\mathrm{TIC}^{36}$.

En el área del cuidado respiratorio, se encontraron algunas aplicaciones en diversos campos, como el de diagnóstico: una que permite registrar, almacenar, reproducir y analizar sonidos respiratorios del paciente ${ }^{37}$; otra para dispositivos móviles Android que hace posible visualizar, analizar y almacenar registros de flujo de aire obtenidos durante pruebas de capacidad pulmonar con espirómetros portátiles ${ }^{38}$; una llamada TERAPP para interpretar gases arterio-venosos ${ }^{39}$, y una aplicación para ambientes simulados de reanimación cardiopulmonar del neonato ${ }^{40}$.

Otras apps se dirigen a la coordinación del paciente con el personal de salud para, por ejemplo, fortalecer la adherencia a tratamientos en enfermedades crónicas como el EPOC ${ }^{41-44}$, identificar y manejar crisis de asma y rinitis alérgica ${ }^{45,46}$, autogestión de la enfermedad e independencia del adolescente con fibrosis quística ${ }^{47}$, la cesación del tabaquismo ${ }^{48}$, entre otras. Ahora bien, en cuanto al cuidado respiratorio del neonato sano, no se encontraron apps diseñadas, por lo cual los artículos relacionados se clasificaron en categorías según su aporte a la atención: aquellas dedicadas al cuidado prenatal, del posparto y del recién nacido, y las dirigidas al cuidado infantil del neonato enfermo o prematuro. Sobre el cuidado general del recién nacido sano, se halló un artículo ${ }^{26}$.

En la atención prenatal y posparto, se encontraron apps diseñadas para acercar los servicios a poblaciones rurales y para acompañar las decisiones de trabajadores comunitarios de salud en cuidado de la gestante y del recién nacido ${ }^{13-20}$. Entretanto, varios artículos describen experiencias con apps de cuidado infantil, principalmente para trabajadores del área de la salud y/o voluntarios en países de bajos ingresos, con resultados que van desde la mejora en la calidad de la atención hasta el impacto en la disminución de la mortalidad infantil ${ }^{21-25}$.

Con respecto al cuidado del lactante sano, se encontró una aplicación sobre nutrición ${ }^{27}$, cuyos resultados sugieren que las características de diseño y entrega de información necesarias para maximizar el impacto de las intervenciones de mHealth en la alimentación infantil aún son inciertas. Cervantes et $a .^{26}$, por su parte, concluyen que la telemedicina es eficaz en el control, seguimiento y prevención de problemas habituales del lactante sano en la 
atención primaria, disminuyendo la frecuencia de visitas a pediatría o a enfermería.

Como se comentaba arriba, esta revisión no encontró apps diseñadas de manera específica para que el cuidador obtenga información sobre signos de alarma o recomendaciones de cuidado respiratorio del recién nacido. Las aplicaciones relacionadas con la atención infantil, al recién nacido o al prematuro, incluían recomendaciones generales, cuyos principales consejos se dirigían a sensibilizar sobre los signos de alerta del recién nacido y sus prácticas de alimentación $^{15}$ y vacunación ${ }^{17}$, así como sobre el seguimiento al desarrollo visual y motor del prematuro ${ }^{24}$, la evaluación neonatal para trabajadores comunitarios $^{25}$ y la lactancia materna ${ }^{26}$.

Si bien el uso de aplicaciones móviles representa ventajas en el ámbito sanitario, acercando los servicios a los pacientes, la calidad de estas apps es muy variable, tanto en contenidos como en funciones, por lo cual la tendencia a futuro será validar su calidad y la seguridad de la información ${ }^{49,50}$. Por último, en cuanto a limitaciones de esta revisión, se debe anotar que solo se incluyeron artículos en revistas indexadas y se exploraron aquellos relacionados con aplicaciones para la salud. En este sentido es preciso considerar que las aplicaciones sobre las que se publica generalmente son derivadas de proyectos de investigación y/o académicos. Por lo demás, no se consideraron otras apps comerciales que pueden existir relacionadas con el tema de interés (cuidado respiratorio).

\section{CONCLUSIÓN}

A pesar de que existen cada día más aplicaciones para móviles relacionadas con salud, no se encontraron herramientas de este tipo enfocadas en el área del cuidado respiratorio del neonato o que fueran específicas para cuidadores. Las más relacionadas se dirigen a aconsejar sobre cuidados generales del recién nacido. No obstante, ante el aumento exponencial del diseño de apps, se requiere empezar a evaluar la calidad, popularidad y utilidad de las aplicaciones para el usuario de salud.

\section{DECLARACIÓN SOBRE CONFLICTO DE INTERESES}

Los autores manifiestan no tener conflictos de interés. Este proyecto se desarrolló como opción de grado, en el marco del proyecto financiado como contrapartida en especie por la Fundación Universitaria del Área Andina y con financiación en especie y dinero de la Universidad de Cundinamarca.

\section{CONTRIBUCIÓN DE LOS AUTORES}

Primer autor: trabajo de campo, análisis y redacción.

Segundo autor: recolección de datos, análisis y redacción.

Tercer autor: recolección de datos, análisis y redacción.

\section{REFERENCIAS BIBLIOGRÁFICAS}

1.Canizzaro C, Paldino M. Fisiología y fisiopatología de la adaptación neonatal. Anestesia, Analgesia, Reanimación. 2011; 24(2): 1-24. Disponible en: http://files.sld.cu/anestesiologia/files/2012/06/fisi ologia-adaptacion-neonatal.pdf.

2.García E, García H, Angulo E, Reyes V, Barrera J, Hernández L, et al. Perfil epidemiológico de prematuros con displasia broncopulmonar en tercer nivel de atención. Revista Médica MD. 2017; 8(4): 172-5. Disponible en: https://go.galegroup.com/ps/anonymous?id=GALE \%7CA534023608\&sid=googleScholar\&v=2.1\&it=r\&l inkaccess $=a b s \& i s s n=20078188 \& p=A O N E \& s w=w$.

3.Fehlmann E, Tapia J, Fernández R, Bancalari A, Fabres J, Dapremont I, et al. Impacto del síndrome de dificultad respiratoria en recién nacidos de muy bajo peso de nacimiento: estudio multicéntrico sudamericano. Arch Argent Pediatr. 2010; 108(5): 393-400. Disponible en: https://www.sap.org.ar/docs/publicaciones/archiv osarg/2010/v108n5a04.pdf. 
4.Castro F, Labarrere Y, González G, Barrios Y. Factores de riesgo del Síndrome Dificultad Respiratoria de origen pulmonar en el recién nacido. Rev Cubana Enfermer. 2007; 23(3). Disponible en: http://scielo.sld.cu/scielo.php?script=sci_arttext\&p id=S0864-03192007000300005.

5.Sánchez-Luna M, Pallás-Alonso C, Botet-Mussons F, Echaniz-Urcelay I, Castro-Conde J, Narbona E, et al. Recomendaciones para el cuidado y atención del recién nacido sano en el parto y en las primeras horas después del nacimiento. An Pediatr (Barc). 2009; 71(4): 349-61. Disponible en: https://www.aeped.es/sites/default/files/7recomendaciones_rn_parto_sen.pdf.

6.Ministerio de Salud y Protección Social. Guía de práctica clínica del recién nacido sano. Bogotá: Minsalud; $2013 . \quad$ Disponible en: https://www.minsalud.gov.co/sites/rid/Lists/Bibliot ecaDigital/RIDE/INEC/IETS/GPC_Prof_Sal_RNSano.p df.

7.López-Candiani C. Cuidados del recién nacido saludable. Acta Pediátrica de México. 2014; 35(6): 513-7. Disponible en: https://www.redalyc.org/articulo.oa?id=42364035 0010.

8.Domínguez-Anaya R, Tapia-Caez E, HernándezEscolar J, Castillo- Ávila I. Edad y nivel educativo asociados al conocimiento sobre signos de alarma para infecciones respiratorias en madres adolescentes. Rev Cuid. 2017; 8(2): 1628-37. Disponible en: https://revistacuidarte.udes.edu.co/index.php/cuid arte/article/view/395

9.Ferreira-Guerrero E, Báez-Saldaña $R$, TrejoValdivia B, Ferreyra-Reyes L, Delgado-Sánchez G, Chilián-Herrera $L$, et al. Infecciones respiratorias agudas en niños y signos de alarma identificados por padres y cuidadores en México. Salud Pública Méx. 2013; 55(2): S307-13). Disponible en: https://www.scielosp.org/pdf/spm/2013.v55suppl2 /S307-S313/es.

10.Gómez-Izquierdo D, Zapata-Vázquez R, ÁvalosGarcía M, Reyes-Islas G. Conocimiento, práctica del cuidador y factor pronóstico de infecciones respiratorias agudas en niños. Horizonte sanitario. 2019; 17(2): 123-9. Doi: http://http://dx.Doi.org/10.19136/hs.a17n2.2047. 11.Abaza, H, Marscholleck M. mHealth Application Areas and Technology Combinations. A Comparison of Literature from High and Low/Middle Income Countries. Methods Inf Med. 2017; 56(7): 105-22. Doi: http://dx.Doi.org/10.3414/ME17-05-0003.

12.Fernández M. Evaluación de apps sanitarias para las mujeres gestantes de Atención Primaria en la consulta de la matrona. Matronas Hoy. 2017; 1(5): 29-37. Disponible en: https://www.enfermeria21.com/revistas/matronas /articulo/108/evaluacion-de-apps-sanitarias-paralas-mujeres-gestantes-de-atencion-primaria-en-laconsulta-de-la-matrona/.

13.Van den Heuvel J, Groenhof T, Veerbeek J, Van Solinge W, Lely AT F, Franx, A, et al. eHealth as the Next-Generation Perinatal Care: An Overview of the Literature. J Med Res. 2018; 20(6). Doi: http://dx.Doi.org/10.2196/jmir.9262.

14. Halili L, Liu R, Hutchinson K, Semeniuk K, Redmann L, Adamo K. Development and pilot evaluation of a pregnancy-specific mobile health tool: a qualitative investigation of SmartMoms Canada. BMC Med Inform Decis Mak. 2018; 18(95). Doi: http://dx.Doi.org/10.1186/s12911-018-0705-8.

15.Chowdhury M, Shiblee S, Jones H. Does mHealth voice messaging work for improving knowledge and practice of maternal and newborn healthcare? BMC Med Inform Decis Mak. 2019; 19(179). Doi: http://dx.Doi.org/10.1186/s12911-019-0903-z.

16.Mbuthia F, Reid $M$, Fichardt A. mHealth communication to strengthen postnatal care in rural areas: a systematic review. BMC Pregnancy Childbirth. 2019; 19(206). Doi: http://dx.Doi.org/10.1186/s12884-019-2531-0.

17.Prinja S, Nimesh R, Gupta A, Bahuguna P, Gupta $M$, Thakur J. Impact of $m$-health application used by community health volunteers on improving utilisation of maternal, new-born and child health care services in a rural area of Uttar Pradesh, India. 
Trop Med Int Health. 2017; 22(7): 895-907. Doi: http://dx.Doi.org/10.1111/tmi.12895.

18. Willcox M, Moorthy A, Mohan D, Romano K, Hutchful D, Mehl G, et al. Mobile Technology for Community Health in Ghana: Is Maternal Messaging and Provider Use of Technology Cost-Effective in Improving Maternal and Child Health Outcomes at Scale? J Med Res. 2019; 21(2). Doi: http://dx.Doi.org/10.2196/11268.

19.Lee S, Nurmatov B, Nwaru I, Mukherjee M, Grant $\mathrm{L}$, Pagliari C. Effectiveness of mHealth interventions for maternal, newborn and child health in low- and middle-income countries: Systematic review and meta-analysis. Journal of Global Health. 2016; 6(1). Doi: https://Doi.org/10.7189/jogh.06.010401.

20.Martínez-Fernández A, Lobos-Medina I, DíazMolina C, Chen-Cruz M, Prieto-Egido I. TulaSalud: An $\mathrm{m}$-health system for maternal and infant mortality reduction in Guatemala. J Telemed Telecare. 2015; 21(5): 283-291. Doi: http://dx.Doi.org/10.1177/1357633X15575830.

21.Bakibinga $P$, Kamande $E$, Omuya $M$, Ziraba A, Kyobutungi $C$. The role of a decision-support smartphone application in enhancing community health volunteers' effectiveness to improve maternal and newborn outcomes in Nairobi, Kenya: quasi-experimental research. BMJ Open. 2017; 7(7): e014896. Doi: http://dx.Doi.org/10.1136/bmjopen2016-014896.

22.Vialart-Vidal MN, Sarduy-Domínguez, Y, DelgadoRamos A, Fleitas-Estevez I. Aplicación de la eSalud en el contexto cubano. Rev Panam Salud Pública. 2018; 42. Doi: http://dx.Doi.org/10.26633/RPSP.2018.19. 23. Matin S, Wallingford A, Xu S, Ng N, Ho A, Vanosdoll $M$, et al. Feasibility of a Mobile Health Tool for Mothers to Identify Neonatal Illness in Rural Uganda: Acceptability Study. JMIR Mhealth Uhealth. 2020; 8(2): e16426. Doi: http://dx.Doi.org/10.2196/16426.

24.Sgandurra G, Lorentzen J, Inguaggiato $E$, Bartalena $L$, Beani $E$, Cecchi $F$, et al. A randomized clinical trial in preterm infants on the effects of a home-based early intervention with the 'CareToy
System". PLoS One. 2017; 12(3): e0173521. Doi: https://doi.org/10.1371/journal.pone.0173521

25. Schaeffer L, Ahmed S, Rahman M, Whelan R, Rahman S, Roy A, et al. Development and evaluation of a mobile application for case management of small and sick newborns in Bangladesh. BMC Med Inform Decis Mak. 2019; 19(116): 12. Doi: http://dx.Doi.org/10.1186/s12911-019-0835-7.

26.Cervantes-Guijarro C, Sánchez-Luna M, BazánFernández $P$. Uso de las nuevas tecnologías y telemedicina en el seguimiento del recién nacido sano. Rev Pediatr Atención primaria. 2014; 16(64): 305-10. Doi: http://dx.Doi.org/10.4321/S113976322014000500005.

27.Russell C, Denney-Wilson E, Laws R, Abbott G, Zheng $M$, Lymer $S$, et al. Impact of the Growing Healthy mHealth Program on Maternal Feeding Practices, Infant Food Preferences, and Satiety Responsiveness: Quasi-Experimental Study. JMIR Mhealth uHealth. 2017; 6(4): e77. Doi: http://dx.Doi.org/10.2196/mhealth.9303.

28.Aguado J, Martínez I, Cañete-Sanz L. Tendencias evolutivas del contenido digital en aplicaciones móviles. profesional de la información. 2015; 24(6): 787-95.

Doi: http://dx.Doi.org/10.3145/epi.2015.nov.10.

29.Valarezo-Pardo M, Honores-Tapia J, GómezMoreno A, Vinces-Sánchez L. Comparación de tendencias tecnológicas en aplicaciones web. 3C Tecnología. Glosas de Innovación aplicadas a las Pymes. 2018; 7(3): 28-49. Doi: http://dx.Doi.org/10.17993/3ctecno.2018.v7n3e27. $28-49 /$.

30.Díaz I, Cáceres Reche M, Trujillo Torres J, Romero Rodríguez J. Impacto de las apps móviles en la actividad física: un meta-análisis. Retos. 2019; 36: 52-7. Disponible en: https://digibug.ugr.es/bitstream/handle/10481/58 990/66628-218120-1-

PB.pdf?sequence $=1 \&$ isAllowed $=\mathrm{y}$.

31.Lee M, Lee H, Kim Y, Kim J, Cho M, Jang J, et al. Mobile App-Based Health Promotion Programs: A Systematic Review of the Literature. Int J Environ 
Res Public Health. 2018; 15(12). Doi: http://dx.Doi.org/10.3390/ijerph15122838.

32.Saigi F, Novillo, D, Piette J. Red CYTED - RITMOS: hacia la búsqueda de soluciones para fomentar la salud móvil en América Latina. Rev Pan Salud Pública. 2017; 41: 1-5. Doi: http://dx.Doi.org/10.26633/RPSP.2017.33.

33.World Health Organization. eHealth, nota descriptiva. WHO; 2019. Disponible en: https://www.who.int/ehealth/about/en/.

34. Kao C, Liebovitz D. Consumer Mobile Health Apps: Current State, Barriers, and Future Directions. PM\&R. 2017; 17(55): S106-15. Doi: http://dx.Doi.org/10.1016/j.pmrj.2017.02.018.

35.Linares-del Rey M, Vela-Desojo L, Cano-de la Cuerda R. Mobile phone applications in Parkinson's disease: A systematic review. Neurología (english edition). 2019; 34(1): 38-54. Doi: https://Doi.org/10.1016/j.nrl.2017.03.006.

36.González-Oñate C, Fanjul-Peyrol C. Aplicaciones móviles para personas mayores: un estudio sobre su estrategia actual. Aula Abierta. 2018; 47(1): 107-12. Doi: https://Doi.org/10.17811/rifie.47.1.2018.107112.

37. Olvera N, Reyes B, Charleston S, González R, Aljama A, Mejía M. Sistema para la detección automática de estertores crepitantes basado en dispositivos móviles inteligentes. Memorias del XL Congreso Nacional de Ingeniería Biomédica. 2017; 4(1): 320-323. México. Disponible en: http://memorias.somib.org.mx/index.php/memori as/article/view/276.

38.Gómez-Chacón A, Díaz-Suárez R, Pabón-Castillo $\mathrm{V}$, Vera-Medina S. Espirómetro electrónico portátil con visualización en dispositivo móvil. Scientia et Technica Universidad Tecnológica de Pereira. 2019; 24(1): $\quad$ 154-60. Doi: http://dx.Doi.org/10.22517/23447214.18451.

39.Cruz-Mosquera F, Herrera-Caballero A, TapiaAngulo $P$, Arango-Arango $A$. Diseño de una aplicación móvil para la interpretación de gases arterio-venosos. Arch Med (Manizales). 2018; 18(1).
Doi:

https://Doi.org/10.30554/archmed.18.1.2583.2018

40. Cavallin F, Binotti M, Ingrassia PL, Genoni G, Rizzollo S, Monzani A, et al. Impact of a mobile application for heart rate assessment in simulated neonatal resuscitation: a randomised controlled cross-over study. Arch Dis Child Fetal Neonatal Ed. 2020; 105(1): 41-4. Doi: http://dx.Doi.org/10.1136/archdischild-2018316757.

41.Yang F, Wang Y, Yang C, Hu H, Xiong Z. Mobile health applications in self-management of patients with chronic obstructive pulmonary disease: a systematic review and meta-analysis of their efficacy. BMC Pulm Med. 2018; 18(147). Doi: http://dx.Doi.org/ 10.1186/s12890-018-0671-z.

42. Rassouli F, Boutellier D, Duss J, Huber S, Brutsche $\mathrm{MH}$. Digitalizing multidisciplinary pulmonary rehabilitation in COPD with a smartphone application: an international observational pilot study. Int J Chron Obstruct Pulmon Dis. 2018; 13: 3831-6.

Doi:

http://dx.Doi.org/10.2147/COPD.S182880.

43.Ambrosino N, Fracchia C. The role of telemedicine in patients with respiratory diseases. Expert Rev Respir Med. 2017; 11(11): 893-900. Doi: http://dx.Doi.org/10.1080/17476348.2017.138389 8.

44.Sleurs K, Seys SF, Bousquet J, Fokkens W, Gorris $S$, Pujin B, et al. Mobile health tools for the management of chronic respiratory diseases. Allergy. 2019; 74(7): 1292-306. Doi: http://dx.Doi.org/10.1111/all.13720.

45.Larenas-Linnemann D, Mullol, J, Ivancevich J, Antó J, Cardona V, Dedeu T, et al. MASK (Mobile Airways Sentinel Network). ARIA's comprehensive solution for mobile app for the multimorbidity of allergic. Rev Alerg Mex. 2019; 66(1): 140-6. Doi: http://dx.Doi.org/10.29262/ram.v66i1.578.

46.Tan R, Cvetkovski B, Kritikos V, O'Hehir R, Lourenço $\mathrm{O}$, Bousquet J, Bosnic-Anticevich $\mathrm{S}$. Identifying an effective mobile health application for 
the self-management of allergic rhinitis and asthma in Australia. Journal of Asthma. 2020; 57(10): 112839.

Doi: http://dx.Doi.org/10.1080/02770903.2019.164072

8.

47.Rudolf I, Pieper K, Nolte H, Junge S, Dopfer C, Sauer-Heilborn A, et al. Assessment of a Mobile App by Adolescents and Young Adults with Cystic Fibrosis: Pilot Evaluation. JMIR Mhealth Uhealth. 2019; 7(11): e12442. Doi: http://dx.Doi.org/10.2196/12442.

48. Alcántara G, Bambs C. Nuevas tecnologías en el tratamiento del tabaquismo. Revista Chilena de Enfermedades Respiratorias. 2017; 33(3): 201-3. Doi: $\quad$ http://dx.Doi.org/10.4067/s071773482017000300201.

49.Alonso-Arévalo J, Mirón-Canelo J. Aplicaciones móviles en salud: potencial, normativa de seguridad y regulación. Rev Cuba Inf Cienc Salud. 2017; 28(3). Disponible en: http://scielo.sld.cu/scielo.php?pid=\$230721132017 000300005\&script=sci_arttext\&tlng=pt.

50.Van der Kleij R, Kasteleyn J, Meijer E, Rianne M, Van der Kleij J, Marise J, et al. SERIES: eHealth in primary care. Part 1: Concepts, conditions and challenges. Eur J Gen Pract. 2019; 25(4): 179-89. Doi: https://doi.org/10.1080/13814788.2019.1658190 